\title{
PROPOSTA DE ENSINO DE COORDENADAS POLARES COM AUXÍLIO DO SOFTWARE GRAFEQ
}

\section{ARTIGO ORIGINAL}

RAMOS, Gilberto Pereira ${ }^{1}$

RAMOS, Gilberto Pereira. Proposta de Ensino de Coordenadas Polares com auxílio do Software GrafEq. Revista Científica Multidisciplinar Núcleo do Conhecimento. Ano 05, Ed. 09, Vol. 04, pp. 35-75. Setembro de 2020. ISSN: 24480959, Link de acesso: https://www.nucleodoconhecimento.com.br/educacao/coordenadas-polares

\section{RESUMO}

Esse trabalho tem como objetivo apresentar uma proposta para a introdução e exploração dos principais conceitos presentes no estudo de coordenadas polares, que faz parte do conteúdo do curso de Matemática do Ensino Médio conforme os PCNs. Esta proposta foi elaborada a partir da constatação, através de estudos preliminares, da existência de dificuldades relativas à compreensão e aprendizagem dos conceitos associados a este tema. Tendo em vista a importância do estudo de coordenadas polares no currículo escolar e na compreensão de fenômenos naturais e nas diversas áreas das ciências, as atividades que compõem esta proposta estão fundamentadas nas ideias de que a aprendizagem se dá através de um processo de construção do conhecimento. Além disso, acreditando que a contextualização dos conteúdos contribui para a aprendizagem significativa da matemática, esse trabalho propõe uma sequência de atividades que, a partir de problemas reais, pretende explorar os principais conceitos presentes no estudo do sistema de coordenadas polares. Para auxiliar os alunos na resolução dos problemas e na exploração destes conceitos,

1 Bacharel em Informática/Ciência da Computação - UFRJ. Licenciado em Matemática - Universidade Estácio de Sá - UNESA - RJ. MBA em Gestão de TI Escola Politécnica da UFRJ. 
utilizamos o software GrafEq. Acreditamos que esta proposta poderá contribuir para a melhoria da qualidade do ensino da matemática e mais especificamente para o ensino de coordenadas polares. Além disso, este trabalho poderá servir como inspiração aos professores de matemática para a criação de novas metodologias de ensino.

Palavras-chaves: GrafEq, ensino, matemática, coordenadas, polares.

\section{INTRODUÇÃO}

O tema software matemático é bastante desafiador e a crescente utilização dos meios computacionais como ferramenta para educação matemática o tornam de grande relevância para o processo ensino-aprendizagem nos dias atuais. Além desse fator motivador, também fui levado a pensar no tema ao cursar a disciplina Informática na Educação, uma das cadeiras do currículo do curso de Matemática da Universidade Estácio de Sá, onde foram apresentadas algumas ferramentas de auxílio ao ensinoaprendizagem da matemática. Ao pesquisar mais sobre o tema descobri a ferramenta GrafEq, que auxilia na tratativa visual de conceitos e estruturas algébricas matemáticas. Tive o interesse em trabalhar o tema 'coordenadas polares' por ele ainda ser pouco presente nas aulas do Ensino médio e por não ter encontrado nas minhas pesquisas a relação entre o conteúdo proposto e a ferramenta adequada. Sendo assim introduzo esse trabalho tomando com impulso essa abordagem de ensino e ao fato dessa proposta de ensino estar adequada as orientações dos PCNs.

O Ministério da Educação (MEC) através do documento intitulado "Parâmetros curriculares nacionais - Ensino Médio (PCNEM) - Volume 2", Capítulo 3, orienta professo res e escolas como tratar os conteúdos matemáticos, metodologias a serem utilizadas, uso de tecnologia, organização curricular e projeto político-pedagógico.

Com isso iremos propor alguns conteúdos que podem ser desenvolvidos com objetivo de complementar a formação matemática dos estudantes. Após pesquisas em bases de artigos de Educação Matemática, como Bolema e Revemat, e na Internet, não foram encontradas abordagens de coordenadas polares com utilização do software 
matemático GrafEq versão 2.13, o que serviu de fator motivador para elaboração desse trabalho.

\section{COMO FAVORECER O PROCESSO ENSINO-APRENDIZAGEM EM} SALA DE AULA?

A pergunta que gera a motivação desse trabalho é a seguinte: "Dada a dificuldade de aprendizagem do conteúdo matemático "transformação de sistemas de coordenadas cartesianas em sistema de coordenadas polares", de que modo podemos favorecer o processo de ensino-aprendizagem em sala de aula?" O presente trabalho parte da premissa que existe uma dificuldade na aprendizagem do conteúdo matemático algébrico no que tange as transformações do sistema cartesiano em sistemas de coordenadas polares, e busca propor uma abordagem que visa facilitar esse aprendizado e que pretende ser respondida nesse trabalho. Esse tema embora previsto nos PCNs ainda está ausente nas aulas do Ensino Médio, vindo aparecer somente na universidade nos primeiros períodos das cadeiras de ciências exatas e afins. Efetuar transformações sempre foi uma problemática no ensino da matemática, pois uma vez que o aluno se acostuma com um universo, a matemática vem e o leva para outro com mais possibilidades.

O aluno é desafiado a ampliar a abstração, é conduzido a novidade e isso gera desconforto. O conceito de transformações do sistema de coordenadas é amplamente usado na matemática e forma a base de sistemas computacionais, com destaque para Álgebra Linear em Computação Gráfica.

Para busca da resposta dessa questão esse trabalho efetua uma pesquisa bibliográfica tendo como base teórica as teorias de aprendizagem construtivistainteracionista, o uso de softwares educacionais como recurso didático e por fim propõe o uso em sala de aula do software matemático GrafEq 2.13 como auxílio no ensino do conteúdo de sistemas de coordenadas polares. 


\section{EDUCAÇÃO MATEMÁTICA E TECNOLOGIA COMPUTACIONAL}

É uma verdade dizer que as tecnologias de informação e comunicação tem sido introduzidas na educação de forma crescente, isso vem a exigir dos alunos uma nova visão e postura nos modos de conceber e trabalhar com os problemas propostos, requerendo destes, novas estratégias cognitivas para solucionar um problema. Fazse necessário também mencionar a importância da formação do professor nesse processo. Para Valente (1999, p. 19) "a questão da formação do professor mostra-se de fundamental importância no processo de introdução da informática na educação, exigindo soluções inovadoras e novas abordagens que fundamentam os cursos de formação".

Stahl (2008, p. 307), menciona que "a educação exige uma abordagem diferente em que o componente tecnológico não pode ser ignorado". Os professores precisam entender que a entrada da sociedade na era da informação exige habilidades que não têm sido desenvolvidas na escola, e que a capacidade das novas tecnologias de propiciar aquisição de conhecimento individual e independente implica num currículo mais exigível, desafia o currículo tradicional e a filosofia educacional predominante, e depende deles a condução das mudanças necessárias (STAHL, 2008).

Segundo Papert (1994), o ensino de matemática, pode liberar-se da pedagogia do treinamento e voltar-se para a formação do homem enquanto ser pensante e criador. O software matemático surge como proposta de um meio complementar para o processo de ensino-aprendizagem.

Segundo Vieira (1999), os softwares educativos podem ser classificados, também, quanto ao nível de aprendizagem do aluno como:

- sequencial - onde objetiva a transferência de informação, apresentação de conteúdos e postura passiva do aluno,

- relacional - onde objetiva a aquisição de certas habilidades, possibilita que o aluno relacione com outros fatos ou outras informações, 
- criativo - onde está relacionado com a criação de novos esquemas mentais, possibilitando haver a interação entre pessoas e tecnologia, postura mais participativa e ativa do aluno.

A educação matemática é fortemente influenciada pelos avanços tecnológicos, uma vez que os recursos de tic podem auxiliar na dificuldade de aprendizagem dos alunos além de propor uma nova dinâmica no ensino da matemática. Os alunos em geral apresentam dificuldades em relação ao processo de aprendizagem, muitas vezes originado pela falta de motivação ou por aulas sem relação com o cotidiano. Considerando tais fatores, este trabalho analisou o uso de software matemático GrafEq 2.13 como auxílio na aprendizagem de matemática tendo como proposta de conteúdo o conceito de coordenadas polares, apresentando-o como uma alternativa para tornar as aulas mais agradáveis aos alunos.

Nas palavras de Baranauskas et al. (1999) a tecnologia computacional tem mudado a prática de quase todas as atividades, das científicas às de negócio até às empresariais. E o conteúdo e prática educacionais também seguem essa tendência. Podemos dizer que a criação de sistemas computacionais com fins educacionais tem acompanhado a própria história e evolução dos computadores.

"O grande desafio é encontrar opções que venham a contribuir na superação das dificuldades encontradas por professores e alunos no ensino-aprendizagem da Matemática" (SOUZA, 2001, p. 24).

Pedagogicamente falando, a utilização de ambientes informatizados, empregando-se softwares educativos avaliados previamente pelo professor, acompanhados de uma didática construtiva e evolutiva, pode ser uma solução interessante para os diversos problemas de aprendizagem em diferentes níveis. (MAGEDANZ, 2004)

Para o professor, o uso do software educativo possibilita uma maior apreensão dos conteúdos pelos alunos, Quanto aos softwares educacionais é fundamental que o professor aprenda a escolhê-los em função dos objetivos que pretende atingir e de sua própria concepção de conhecimento e de aprendizagem, distinguindo os que se 
prestam mais a um trabalho dirigido para testar conhecimentos dos que procuram levar o aluno a interagir com o programa de forma a construir conhecimento. (PCN, BRASIL, 1997)

A escolha do software GrafEq 2.13 deveu-se ao fato de este ser um programa gratuito, de fácil acesso, no qual é possível construir gráficos e figuras geométricas utilizando equações, inequações e intervalos numéricos, utilizando coordenadas cartesianas ou polares.

Um fator motivador também foi o fato de ainda não existir artigos publicados relacionando o uso do software matemático GrafEq 2.13 com o conceito de transformações em coordenadas polares. "Nas construções com o GrafEq são necessários os conceitos de função, equação, inequação e suas representações gráficas, [...]" (GRAVINA; BASSO, 2012, p. 113).

Como consequência se torna uma boa alternativa para o processo de ensinoaprendizagem, devido a todas as possibilidades e funções que este programa proporciona. Segundo Valente (1994 e 1999), o uso do computador na educação objetiva a integração deste no processo de aprendizagem dos conceitos curriculares em todas as modalidades e níveis de ensino, podendo desempenhar um papel de facilitador entre o aluno e a construção do seu conhecimento.

O autor defende a importância de se abrir a mente para as novas abordagens computacionais visando melhorar a qualidade das aulas ministradas. Busca também através desse trabalho propor um caminho a ser explorado pelo professor na tratativa visual de conceitos e estruturas matemáticas para melhor apresentação de conteúdo matemático para os alunos.

\section{MATERIAIS E MÉTODOS}

Esse trabalho consiste em uma pesquisa bibliográfica acerca das teorias de aprendizagem que dão suporte ao tema, bem como o estudo investigativo do software matemático GrafEq 2.13, suas potencialidades e sua aplicação no ensino da 
matemática para o conteúdo de sistemas de coordenadas polares. A pesquisa também aborda os meios de se trabalhar com softwares educacionais como recurso didático nas aulas de matemática.

Segundo Fonseca (2002) apud Gerhardt e Silveira (2009),

A pesquisa bibliográfica é feita a partir do levantamento de referências teóricas já analisadas, e publicadas por meios escritos e eletrônicos, como livros, artigos científicos, páginas de web sites. Qualquer trabalho científico inicia-se com uma pesquisa bibliográfica, que permite ao pesquisador conhecer o que já se estudou sobre o assunto. Existem porém pesquisas científicas que se baseiam unicamente na pesquisa bibliográfica, procurando referências teóricas publicadas com o objetivo de recolher informações ou conhecimentos prévios sobre o problema a respeito do qual se procura a resposta (FONSECA, 2002, p. 32 apud GERHARDT e SILVEIRA, 2009, p. 37).

Ao se conjecturar o tema iniciou-se a pesquisa buscando materiais/artigos que pudessem servir de base e referência para o projeto de pesquisa, com isso foi possível identificar quais os pontos importantes a serem trabalhados no decorrer da pesquisa, assim como a estrutura que o trabalho deveria seguir. Isso possibilitou ao aluno pesquisador um aporte teórico, a $\mathrm{m}$ de embasar seu trabalho. A pesquisa bibliográfica fundamentou-se em estudos acerca do uso de softwares educacionais no ensino da matemática.

Dessa forma, foi feita a busca por artigos científicos, dissertações e sites que tratassem do tema em questão. A partir da leitura dos materiais considerados relevantes para o trabalho, foi constituída a fundamentação teórica. Os resultados obtidos serão apresentados em quatro etapas, nas quais iremos a princípio falar sobre as teorias de aprendizagem que dão suporte ao tema, o que são softwares educacionais, em seguida sobre o uso deles como recurso didático no ensino de matemática, e por m, como se trabalhar com o software matemático GrafEq 2.13 para o auxílio no ensino do conteúdo de sistemas de coordenadas polares para o Ensino médio e superior. 
Essa proposta de trabalho e pesquisa pretende estabelecer um plano de ação que favoreça o processo de ensino-aprendizagem em sala de aula para o tema específico "aprendizado de coordenadas polares".

Todas as atividades poderão ser desenvolvidas com algum conhecimento sobre funções, conteúdo exposto no ensino fundamental. Além disso, os conhecimentos também estão relacionados aos conjuntos numéricos, representação de pontos no plano cartesiano e regras básicas de álgebra.

O professor, por sua vez, deverá aprender os principais comandos do software GrafEq, o que poderá ser feito através da leitura de alguns tutoriais disponíveis na internet e que serão referenciados mais adiante.

Sabemos que o a metodologia utilizada em sala de aula para ensinar os conteúdos matemáticos é um dos recursos mais importantes para garantir que o aluno efetivamente os aprenda.

O desenvolvimento da primeira atividade com o uso do GrafEq tem como objetivo construir uma circunferência em ambos os sistemas: cartesiano e polar. $\mathrm{O}$ aluno poderá com esse exemplo ter uma noção intuitiva do sistema polar e verá como é fácil essa transformação.

Para a realização da segunda atividade, propomos outra situação-problema. Nesta situação o objetivo é conhecer a equação geral da espiral e construir o seu gráfico.

A terceira atividade será desenvolvida com o intuito de explorar e introduzir o estudo de uma categoria de curvas denominadas rosáceas, conhecer sua equação geral e construir o seu gráfico.

A quarta atividade apresenta uma categoria de curvas chamada de cardioides, que tem esse nome, por ter um formato de coração. 
Nos dois primeiros problemas, os alunos terão a oportunidade de discutir sobre a representação gráfica das situações e desenvolver desta forma sua criatividade e criticidade.

A seguir, começaremos a explorar a representação gráfica de diferentes tipos de funções em coordenadas polares, com o objetivo de fazer com que o aluno perceba a relação entre a lei de formação e sua representação gráfica, evidenciando a propriedade que caracteriza este tipo de função e alguns aspectos importantes relativos a seus coeficientes.

Ao permitir que o computador gere um gráfico na tela através da ferramenta, e poder modificar esse gráfico quantas vezes quiser e testar possibilidades, a atividade tornase mais agradável e enriquecedora para o aluno, uma vez que possibilita oportunidades de descoberta.

\section{SOBRE O SOFTWARE GRAFEQ}

O software Grafeq é um software que trabalha com equações e inequações, em coordenadas cartesianas e polares. Esse software canadense gera gráficos de equações e inequações de figuras planas com base numa forma generalizada de aritmética intervalar desenvolvida por Jeff Tupper. A "aritmética de Tupper", como é chamada, se encontra magistralmente exposta na sua tese Graphing Equations with Generalized Interval Arithmetic (disponível na sua página pessoal). A utilização do GrafEq ${ }^{4}$ não exige nenhum conhecimento de aritmética intervalar, mas é interessante a leitura do belíssimo trabalho de Tupper como um exemplo importante de como a abstração e a sofisticação matemáticas podem produzir resultados práticos.

\section{UMA PROPOSTA PARA O ENSINO DE COORDENADAS POLARES}

Neste capítulo apresentamos uma sequência didática visando utilizar atividades diferenciadas para a introdução e o desenvolvimento do estudo de coordenadas polares para alunos do $3^{\circ}$ ano do ensino médio. As questões presentes nesta proposta visam garantir que o aluno faça parte do processo de construção do saber. O professor 
com isso tem o papel de conduzir as atividades, e fazê-las de modo a envolver os alunos através de debates e questionamentos, o que, acreditamos, levará ao desenvolvimento escalável dos conceitos que cercam o estudo de coordenadas polares e suas aplicações.

\section{COORDENADAS POLARES}

Conforme trecho retirado do Livro Calculo Vetorial e Geometria Analítica, segundo o autor, temos a seguinte definição para coordenadas polares:

Dado o par ordenado $(a, b)$ de números reais, com $(a, b) \neq(0,0)$, o par $(r$, $\theta)$, com $a=r \cdot \cos (\theta)$ e $b=r \cdot \operatorname{sen}(\theta)$, é dito uma representação de $(a, b)$ em coordenadas polares. Costuma-se dizer, embora $\theta$ esteja definido apenas a menos de um múltiplo inteiro de $2 \pi$, que $r$ e $\theta$ são as "coordenadas polares" de (a,b). (ACKER, 2016, p.103)

Exemplo:

Figura 1 - coordenadas cartesianas e coordenadas polares

\begin{tabular}{|r|r|l|r|r|}
\hline \multicolumn{2}{|c|}{ coordenadas cartesianas } & & \multicolumn{2}{c|}{ coordenadas polares } \\
\hline $\mathrm{a}$ & $\mathrm{b}$ & & $\mathrm{r}$ & ângulo $\left(\theta^{\circ}\right)$ \\
\hline 1 & 0 & & 1,00 & 0 \\
\hline 0 & 2 & & 2,00 & 90 \\
\hline-3 & 0 & & 3,00 & 180 \\
\hline 0 & -3 & & 3,00 & 270 \\
\hline 1 & 1 & & 1,41 & 45 \\
\hline-2 & -2 & & 2,83 & 135 \\
\hline
\end{tabular}

Fonte: O Autor. 
Figura 2 - gráfico exemplo de coordenadas cartesianas

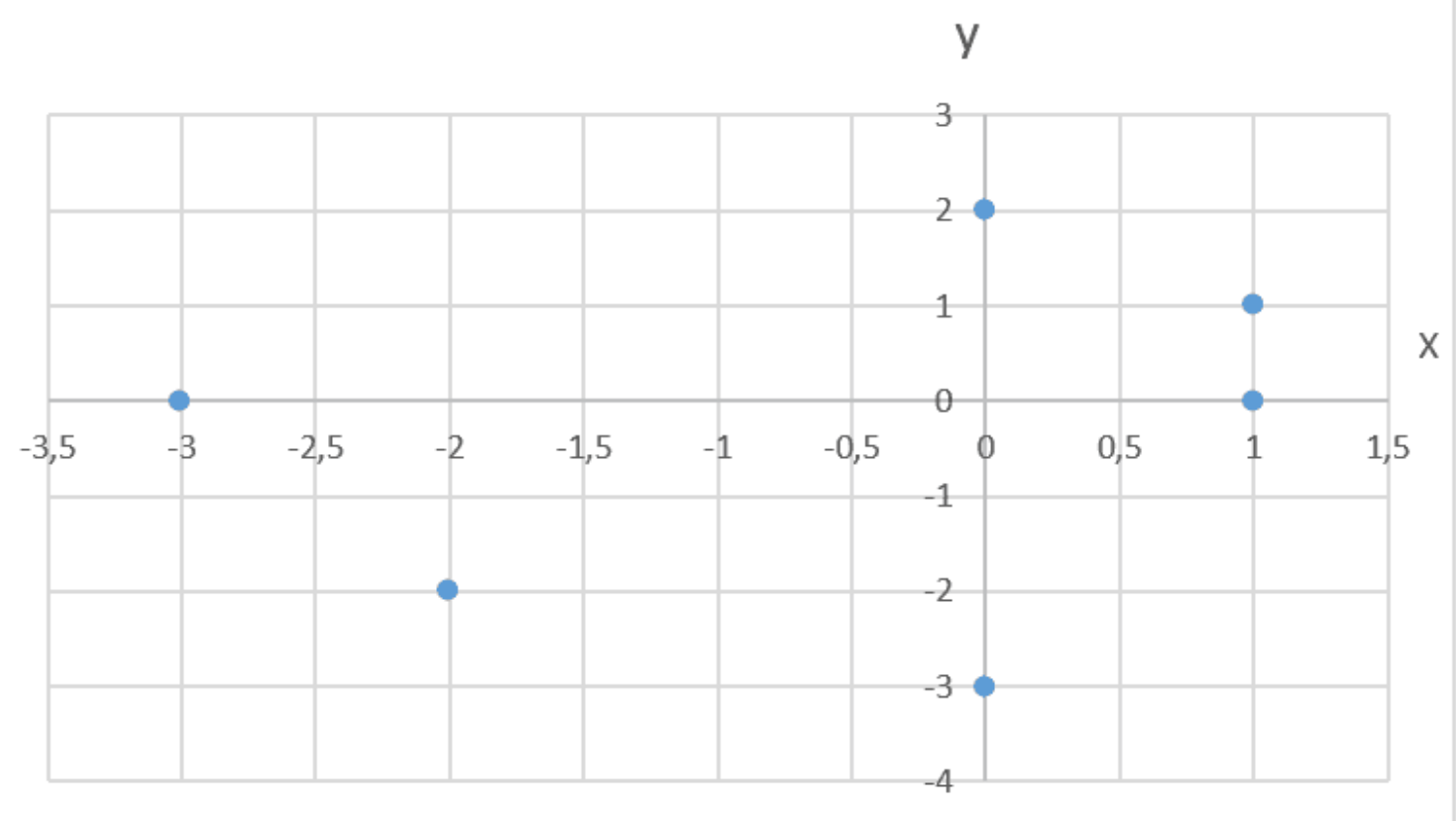

Fonte: O Autor.

Figura 3 - gráfico exemplo de coordenadas polares

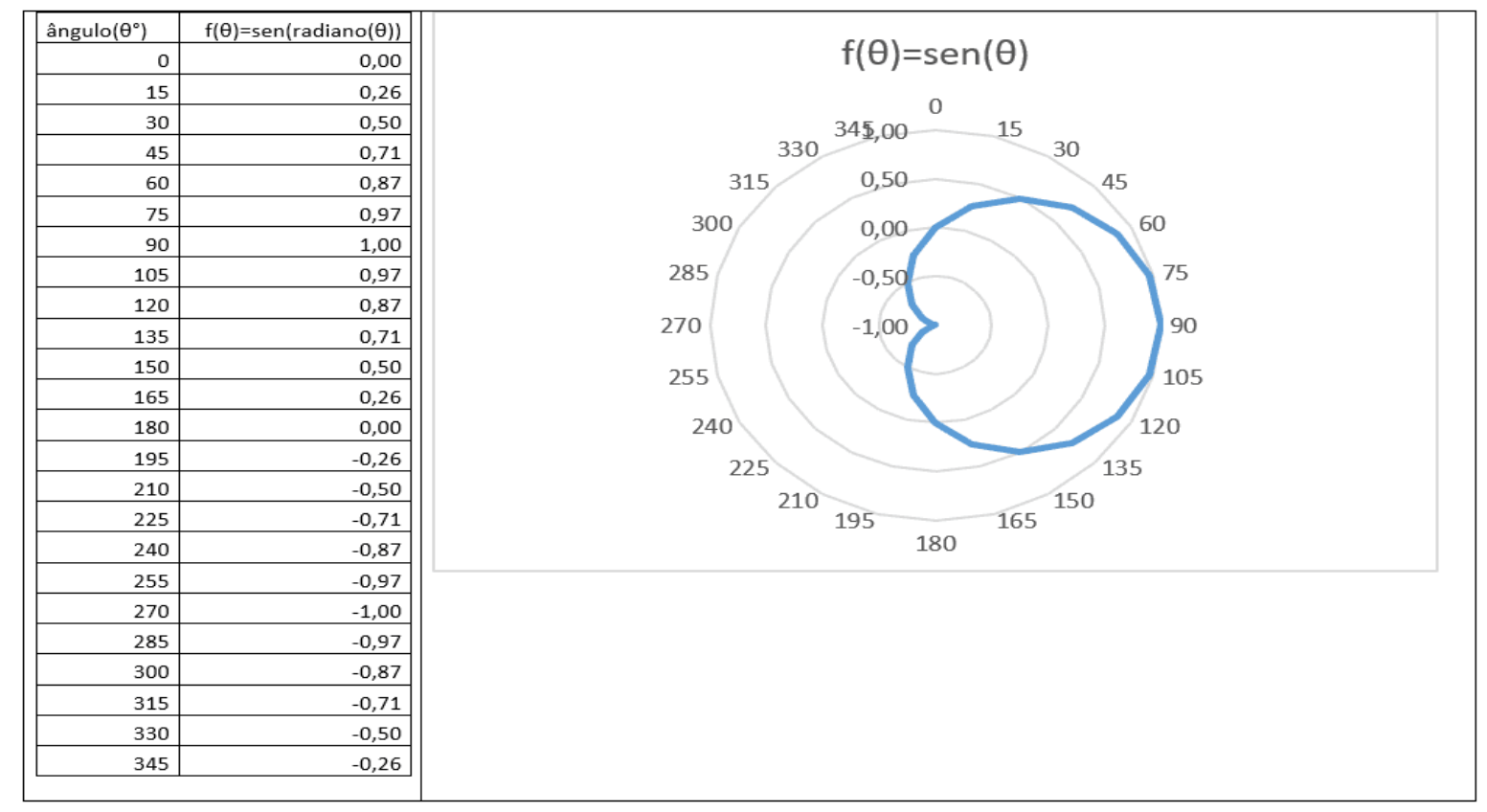

Fonte: O Autor. 
Figura 4 - eixo polar

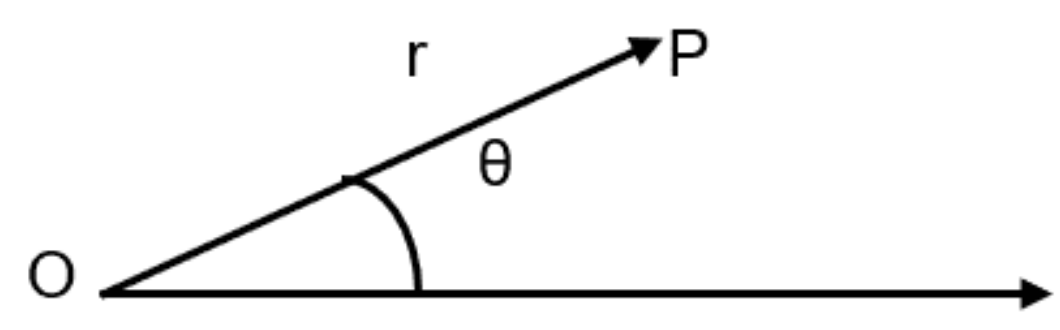

Fonte: O Autor.

Dá-se o nome de polo ao ponto fixo $\mathrm{O}$, e a semirreta, eixo polar.

Vale lembrar que um mesmo ponto $P=(r, \theta)$ não diz respeito apenas a um par ordenado, mas a uma classe de pares ordenados.

Exemplo 1: $(1,-\pi / 4)=(1,7 \pi / 4)$

Figura 5 - exemplo 1

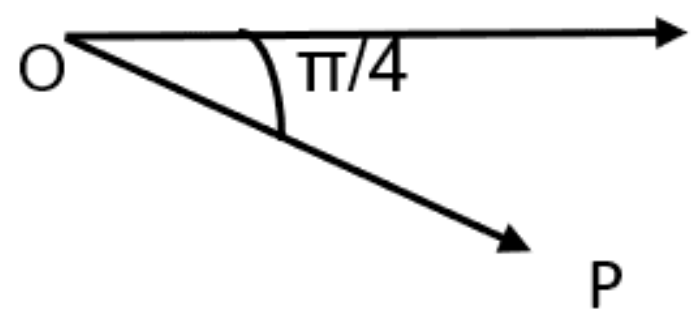

Fonte: O Autor.

Denotamos $P$ por $(-r, \theta)$, para r positivo, se $P=(r, \pi+\theta)$, ou seja, consideramos $(-r, \theta)$ $=(r, \theta+\pi)$. Assim, $(-r, \theta)$ é o simétrico de $(r, \theta)$ em relação ao polo. 
Figura 6 - simetria

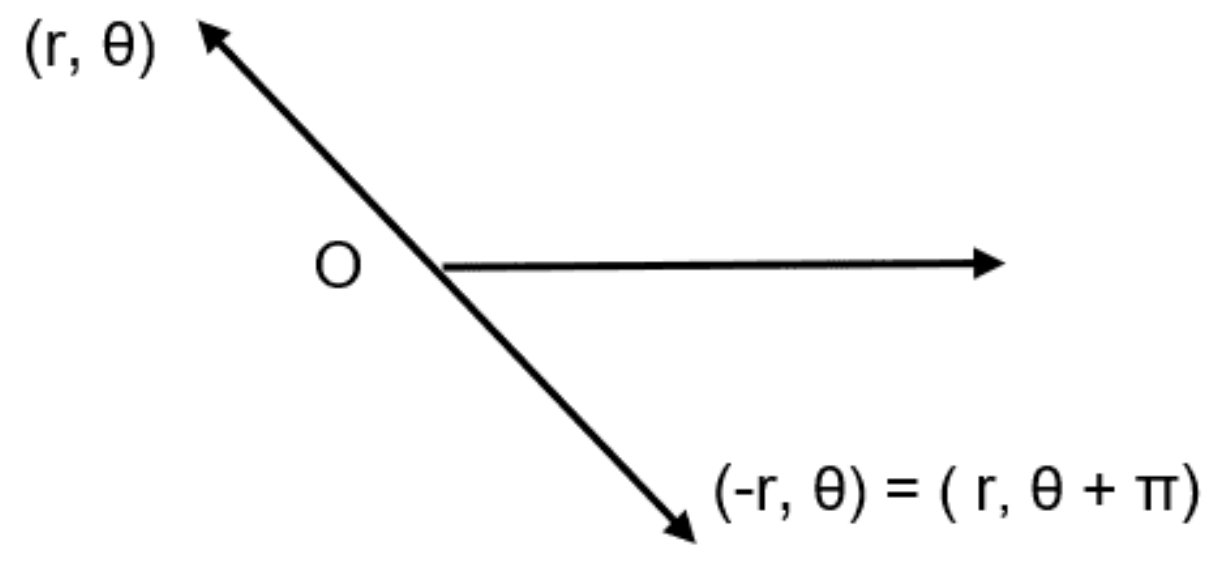

Fonte: O Autor.

Exemplo 2: $(3, \pi / 2)=(-3,3 \pi / 2)$

Figura 7 - exemplo 2

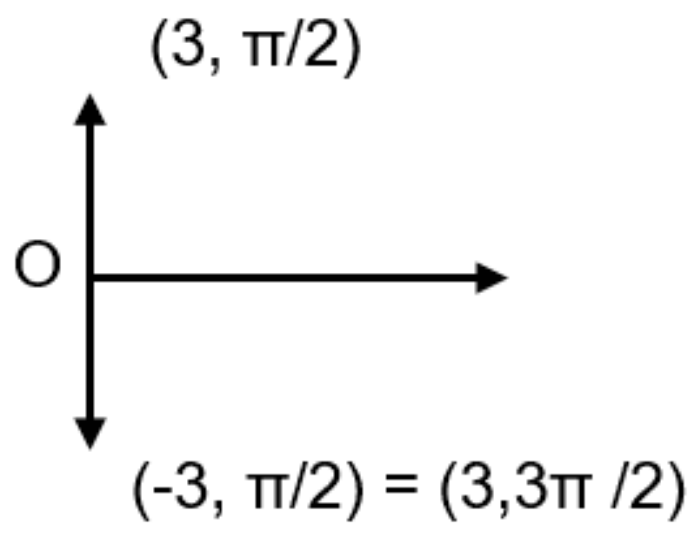

Fonte: O Autor.

Observe que um ângulo $\theta$ pode ser representado por $\theta+2 k \pi$, para todo $k$ inteiro. Assim, $(r, \theta)=(r, \theta+2 \pi)=(r, \theta+4 \pi)=(r, \theta-2 \pi)=(r, \theta-4 \pi)=\ldots$

Exemplo. $(5, \pi / 2)=(5, \quad \pi / 2+10 \pi)=(5,21 \pi / 2)$ 


\section{MUDANÇA DE COORDENADAS}

Consideremos um ponto $\mathrm{P}$ no plano, podemos representar esse ponto em coordenadas cartesianas por $(x, y)$ ou por $(r, \theta)$, em coordenadas polares. Para simplificar, vamos considerar o ponto $\mathrm{O}$ coincidindo com a origem do sistema cartesiano e, a parte não negativa do eixo $\mathrm{x}$, vai representar a semirreta.

1. Efetuando a transformação de coordenadas polares para coordenadas cartesianas.

Dado um ponto $\mathrm{P}$ com coordenadas polares $(r, \theta)$.

Para $0<\theta<\pi / 2$ e $r>0$

Obtemos as seguintes relações no triângulo retângulo OPx.

Figura 8 - mudança de coordenadas

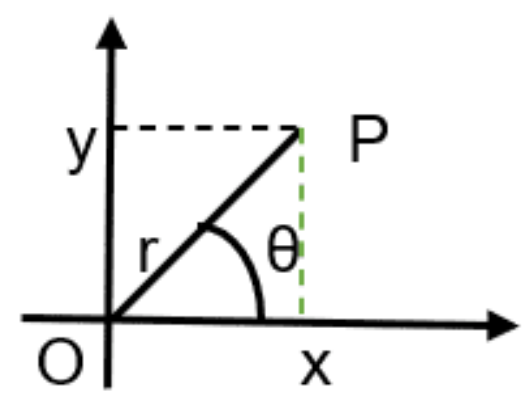

Fonte: O Autor.

$x=r \cdot \cos (\theta)$ e $y=r \cdot \operatorname{sen}(\theta)$

b) Efetuando a transformação de coordenadas cartesianas para coordenadas polares

Dado um ponto $\mathrm{P}$, de coordenadas cartesianas $(\mathrm{x}, \mathrm{y})$. Como vimos acima, considerando $P$ com coordenadas $(r, \theta)$, temos as relações $x=r \cos (\theta)$ e $y=r \operatorname{sen}(\theta)$ 
Como $x^{2}+y^{2}=r^{2} \cos ^{2} \theta+r^{2} \operatorname{sen}^{2} \theta=r^{2}\left(\cos ^{2} \theta+\operatorname{sen}^{2} \theta\right)=r^{2} x 1=r^{2}$, temos que $r=\sqrt{ } x^{2}$ $+\mathrm{y}^{2}$.

Se $r=0$, isto é, $x=y=0$ então podemos tomar qualquer.

Se $r \neq 0, \theta$ é tal que $\cos \theta=x / r$ e $\operatorname{sen} \theta=y / r$.

Exemplo:

Caso $P$ tenha coordenadas polares $(-2, \pi / 6)$, então $x=-2 \cos (\pi / 6)$ e $Y=2-\operatorname{sen}(\pi / 6)$.

Com isso, $x=-1$ e $y=-\sqrt{ } 3$, daí segue que $P$ tem coordenadas cartesianas $(-1,-\sqrt{ } 3)$.

Exemplo:

Com $P$ tendo coordenadas cartesianas $(-1,1)$, então $r^{2}=(-1)^{2}+1^{2}=2$, ou seja, $r=\sqrt{ } 2$.

Como $\cos \theta=-\sqrt{ } 2 / 2$ e $\operatorname{sen} \theta=\sqrt{ } 2 / 2$, então $\theta=3 \pi / 4$.

Assim resulta que $P$ tem coordenadas polares: $(\sqrt{ } 2,3 \pi / 4)$

\section{PRIMEIRA ATIVIDADE}

A primeira atividade de nossa sequência busca apresentar aos estudantes uma ideia intuitiva do conceito de coordenadas polares.

Exemplo. A circunferência de centro na origem e raio 3 tem equação cartesiana $x^{2}+y^{2}$ = 9. Como $x=r \cos (\theta)$ e $y=r \operatorname{sen}(\theta)$, então $r^{2}=9$, ou seja, $r=3$ é a equação polar dessa circunferência.

Utilizando o GrafEq para traçar a circunferência citada em ambos os sistemas de coordenadas:

No sistema de coordenadas cartesiano: 
Figura 9 - Circunferência de raio $r=3$

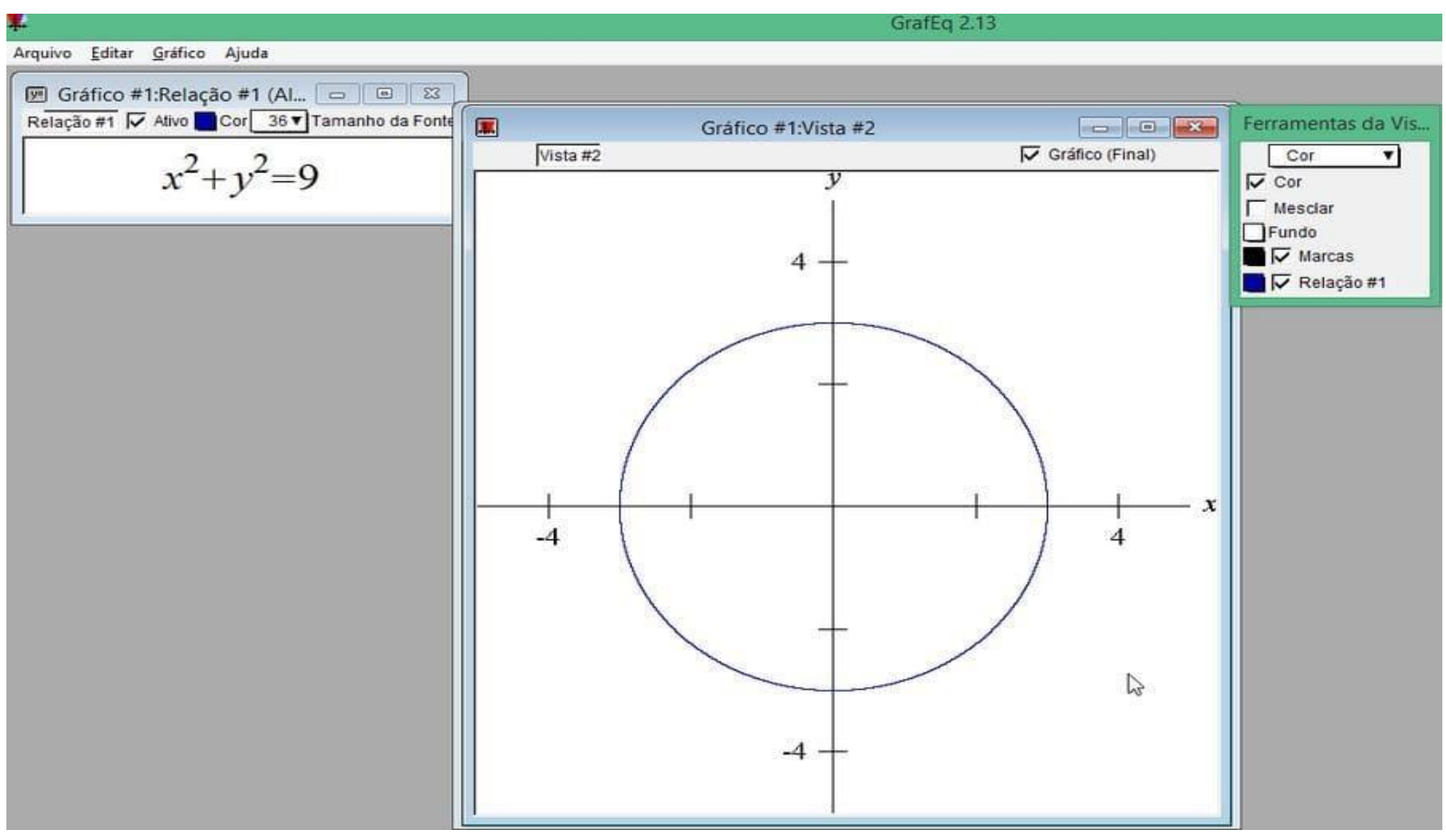

Fonte: O Autor.

Figura 10 - Circunferência de raio $r=3$, em coordenadas polares.

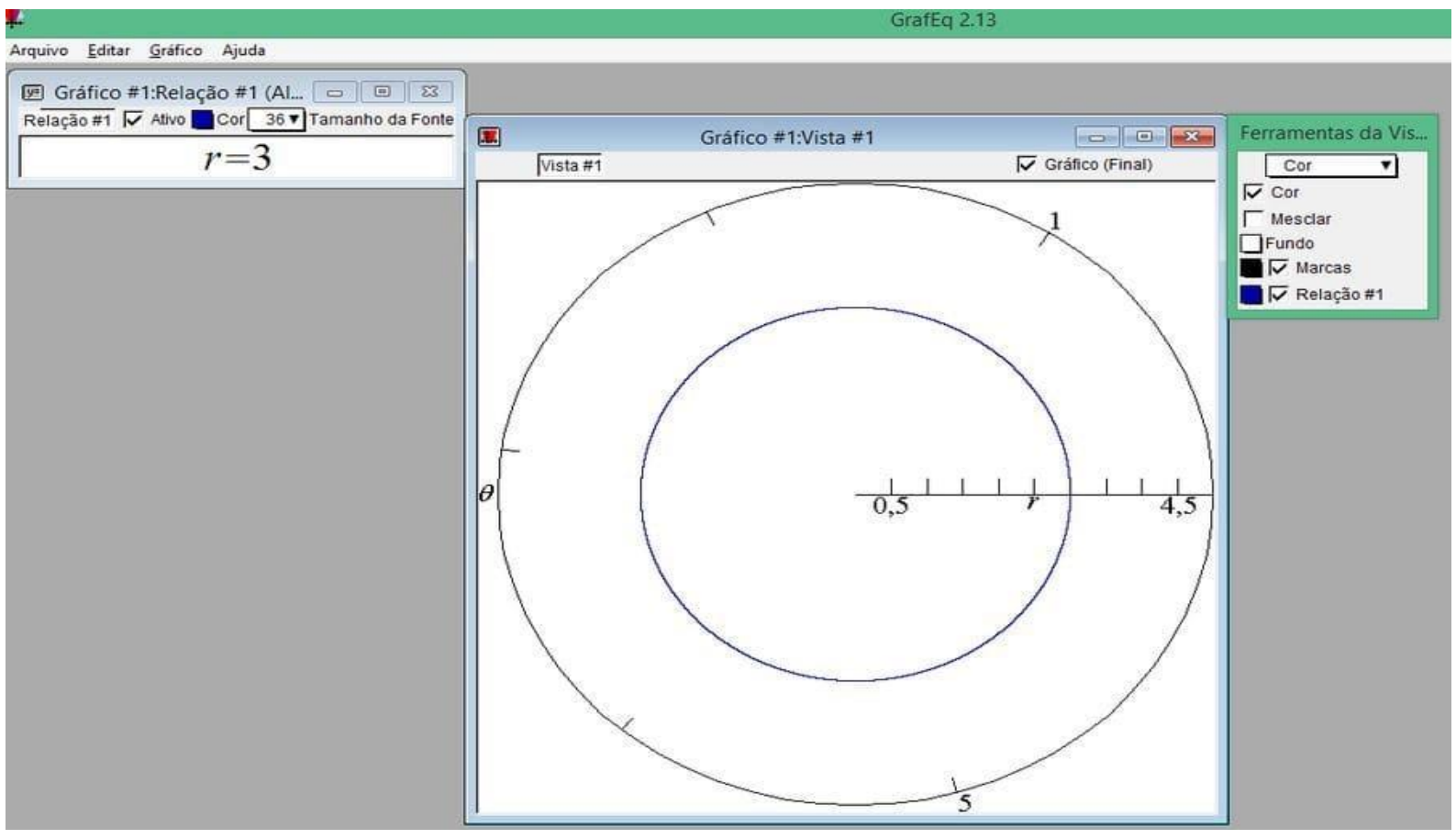

Fonte: O Autor.

RC: 59394

Disponível em: https://www.nucleodoconhecimento.com.br/educacao/coordenadas-polares 


\section{SEGUNDA ATIVIDADE}

Resolva em coordenadas polares o gráfico de $r=\boldsymbol{\theta}, \boldsymbol{\theta} \geq \mathbf{0}$.

O gráfico de $r=\boldsymbol{\theta}$ diz respeito aos pontos $P=(r, r)$ onde $r \geq 0$, ou seja, todos os pontos $\mathrm{P}$, onde temos a distância de $\mathrm{P}$ ao polo igual ao próprio ângulo $\boldsymbol{\theta}$, em radianos, entre o segmento OP e o eixo polar. A equação geral da espiral será dada por $\mathbf{r}=\mathbf{a} \boldsymbol{\theta}$, onde $\theta \geq 0$ e a inteiro.

Usando GrafEq plote os gráficos de $r=\theta$ e $r=-\theta$, para $0 \leq \theta \leq 4 \pi$.

Resposta:

\section{Dica da ferramenta: Configuração do GrafEq para estilo Polar:}

Figura 11-Item de menu Nova Vista

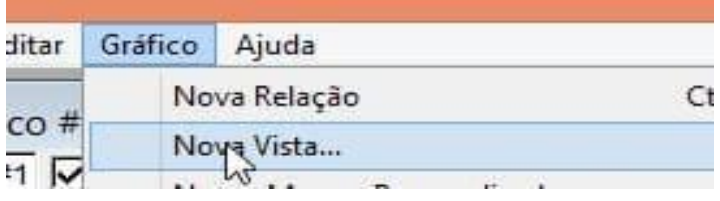

Fonte: O Autor.

Figura 12-Legenda

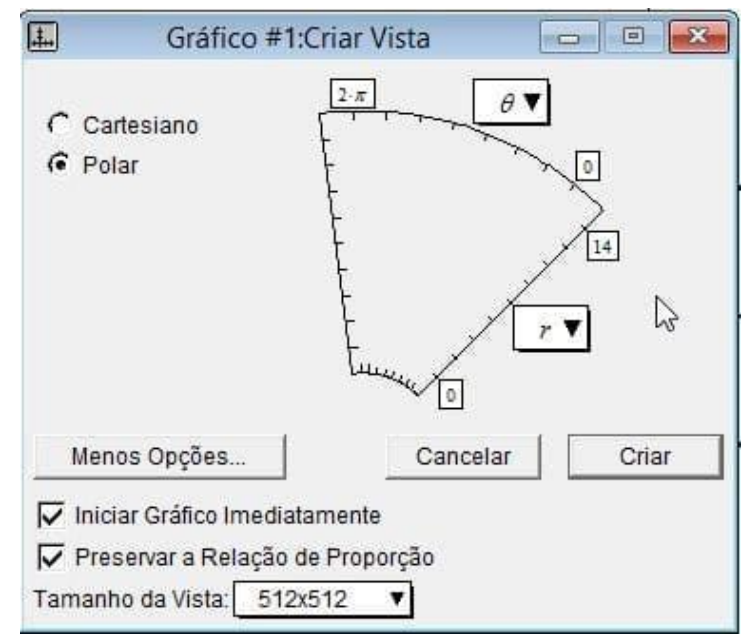

Fonte: O Autor. 
Dica: Duplo click sobre a fórmula abre a caixa de botões rápidos.

Figura 13-Botões rápidos

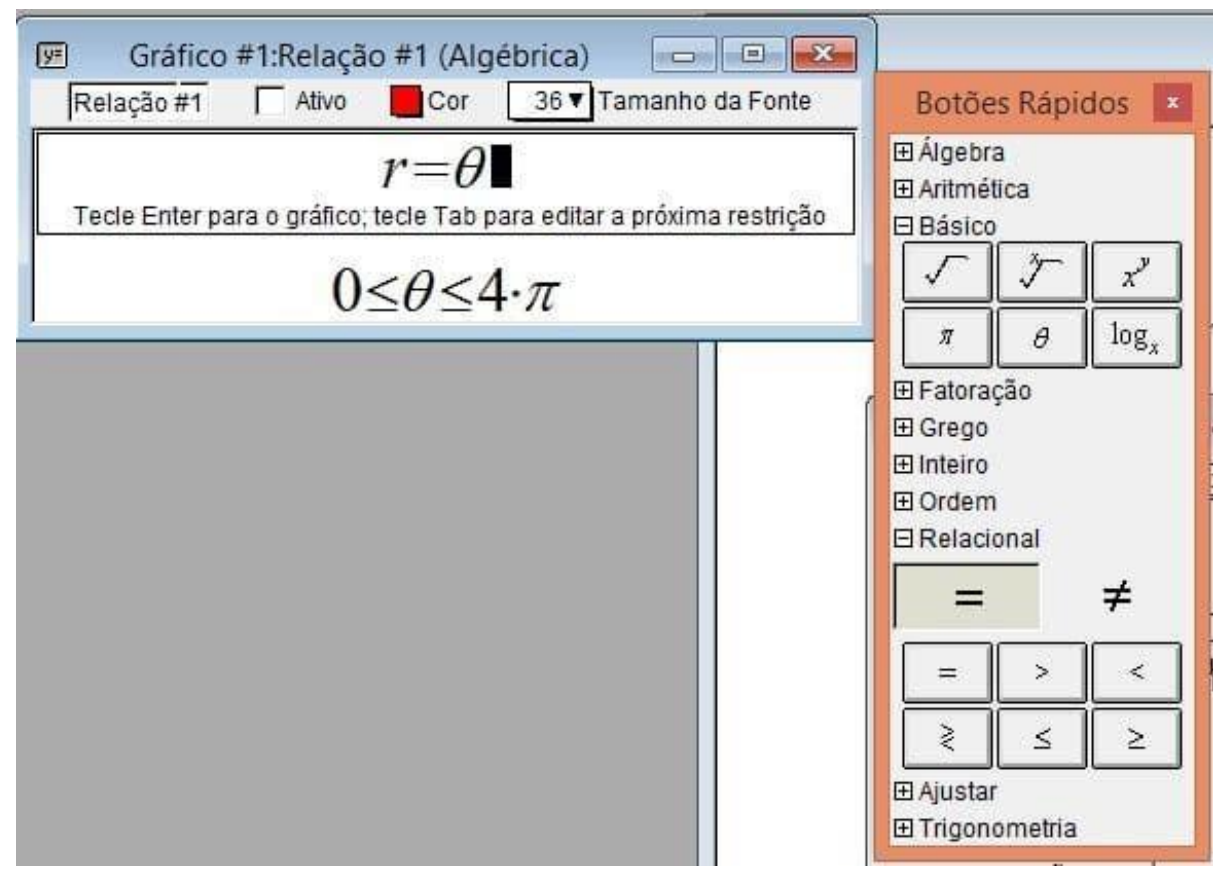

Fonte: O Autor.

Figura 14-Solução 1-a

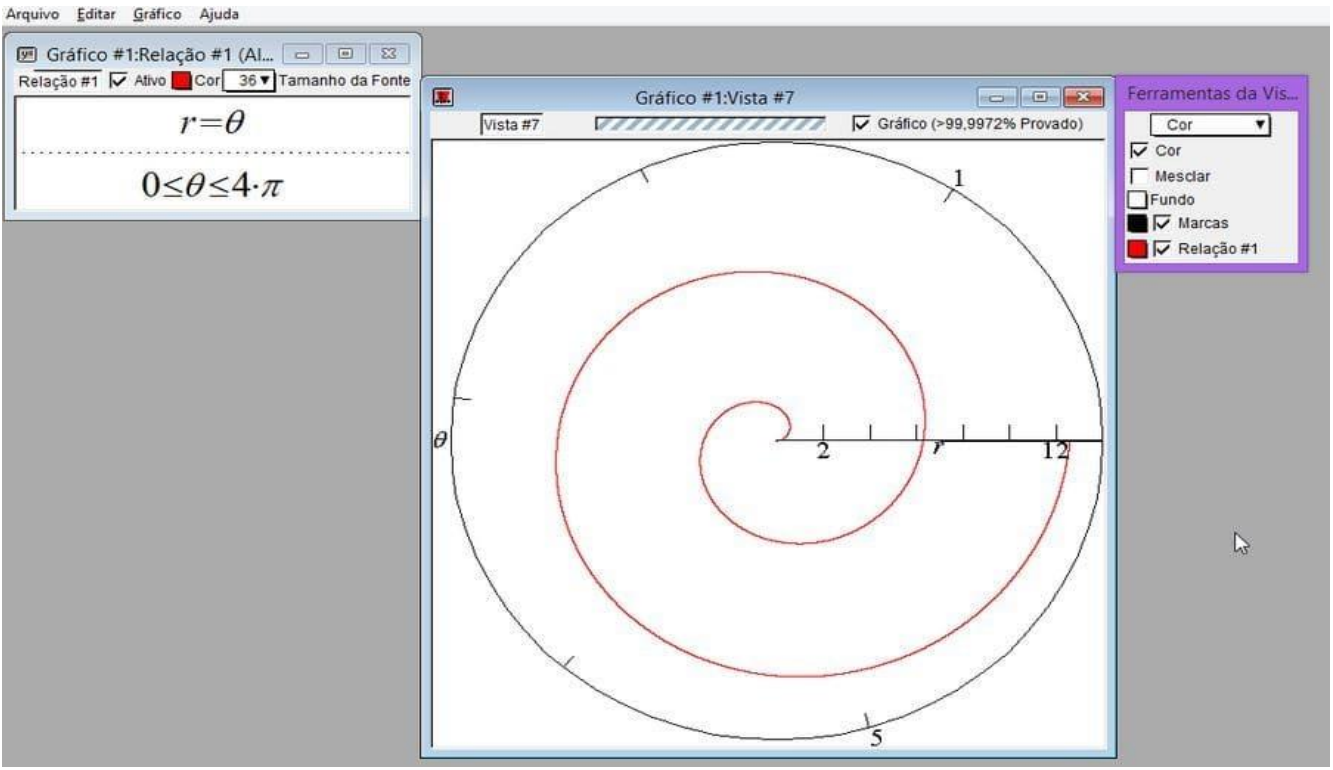

Fonte: O Autor.

RC: 59394

Disponível em: https://www.nucleodoconhecimento.com.br/educacao/coordenadas-polares 
Figura 15-Solução 1-b

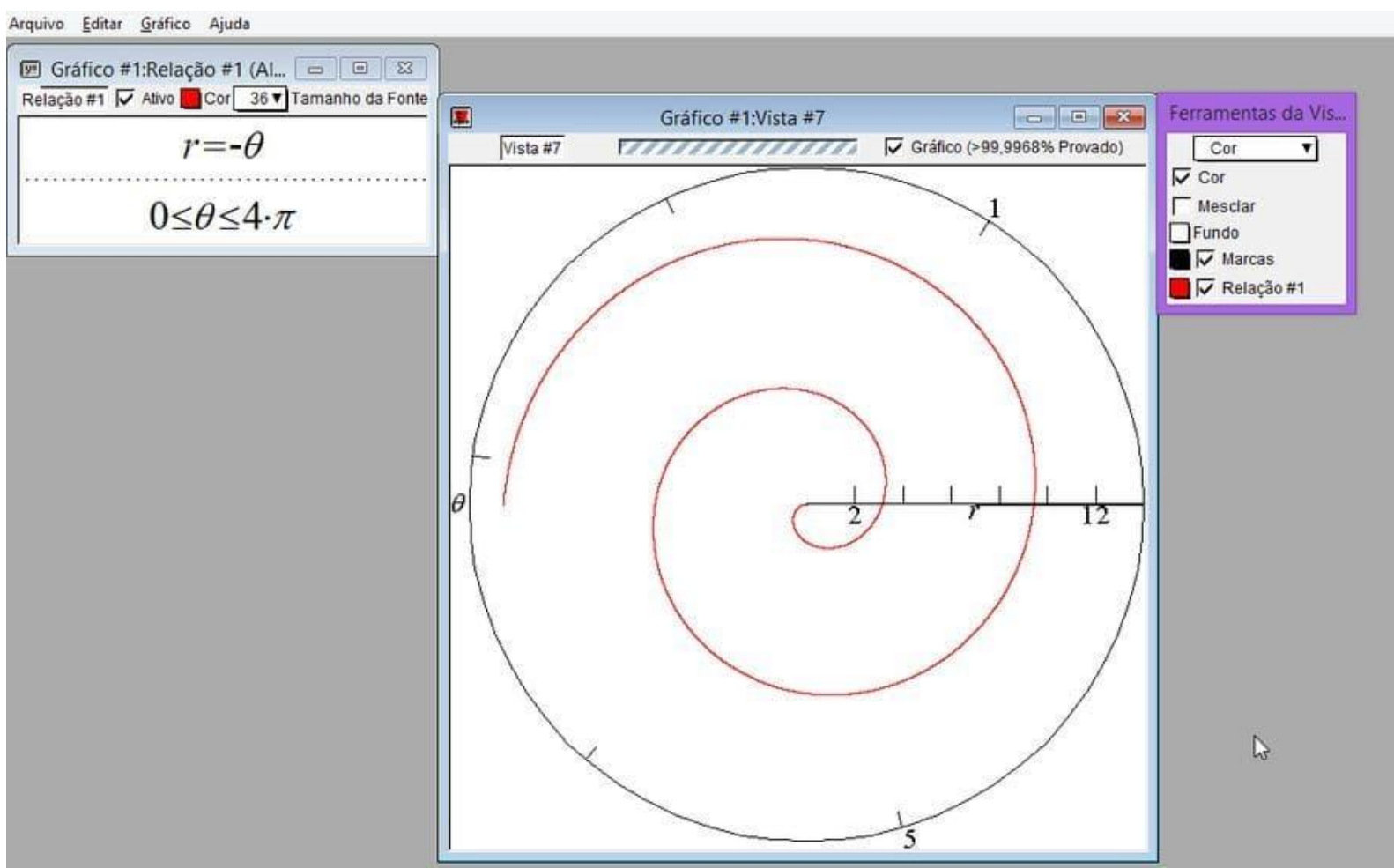

Fonte: O Autor.

\section{TERCEIRA ATIVIDADE}

Usando GrafEq, resolva em coordenadas polares o gráfico de $r=\cos (2 \theta), 0 \leq \theta \leq 2 \pi$.

Solução: 
Figura 16-Rosácea

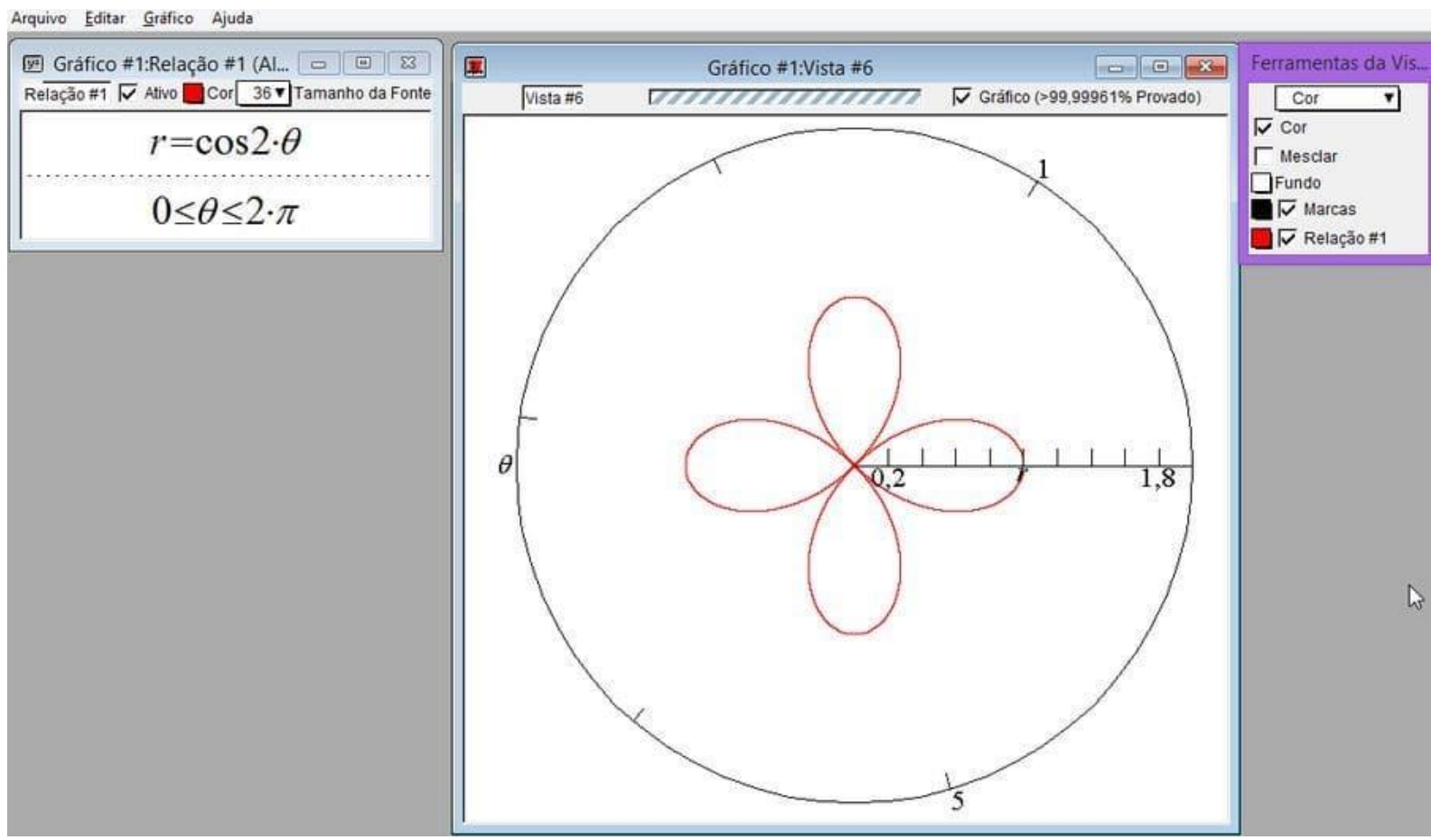

Fonte: O Autor.

Observe que toda equação do tipo $r=\operatorname{asen}(n \theta)$ ou $r=\operatorname{acos}(n \theta)$ para qualquer $n$ inteiro e positivo, representam o conjunto de curvas do tipo rosáceas.

\section{QUARTA ATIVIDADE}

Conhecendo as Cardioides

Usando GrafEq, resolva o gráfico de $r=1+\cos (\theta), 0 \leq \theta \leq 2 \pi$ 
Figura 17-Cardióide

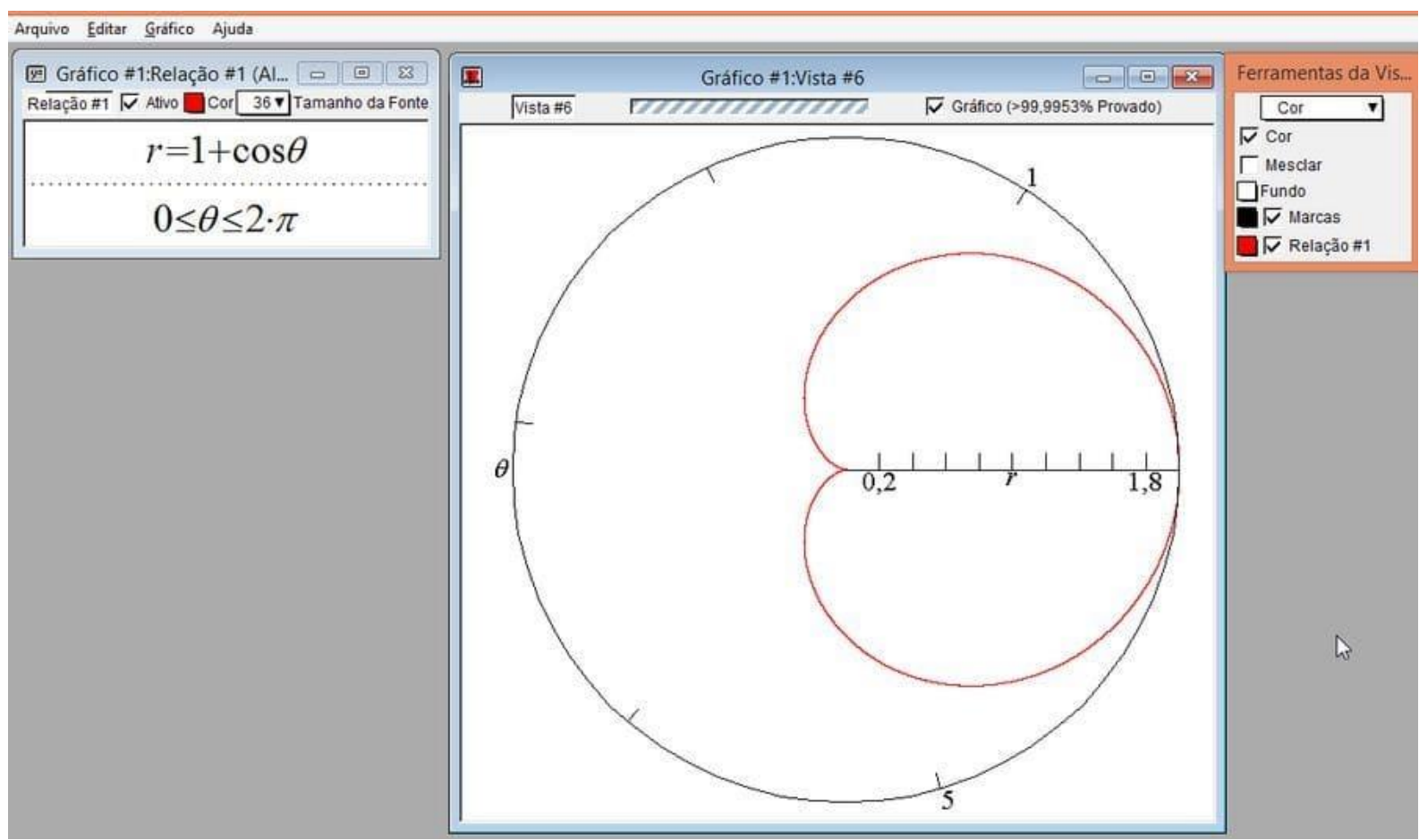

Fonte: O Autor.

Observe que toda equação do tipo $r=a(1+-\operatorname{sen}(\theta))$ ou $r=a(1+-\cos (\theta))$ representam um conjunto de curvas do tipo cardioides, assim chamadas por possuírem a forma de coração.

\section{CONSIDERAÇÕES FINAIS}

O presente trabalho teve como objetivo elaborar uma proposta para a introdução exploração de conceitos presentes no estudo de coordenadas polares com foco no aprendizado de alunos do ensino médio e talvez primeiro ano do ensino superior nas cadeiras de ciências exatas.

Cremos que a proposta aqui apresentada atende a estas necessidades e poderá contribuir para a apropriação do saber matemático por parte dos alunos. Esta crença pode ser fundamentada nas escolhas metodológicas e na abordagem que as atividades foram elaboradas. A escolha do software e a metodologia de resolução de 
problemas possibilitaram uma maior exploração dos aspectos envolvidos no estudo dos sistemas de coordenadas polares de um modo atrativo e significativo.

Esta proposta é uma abordagem para o ensino de coordenadas polares sujeito a correções e adaptações. No decorrer da estruturação das atividades, percebemos que uma simples situação-problema poderia gerar uma grande oportunidade para o desenvolvimento dos conteúdos escolares aqui abordados. De modo que, entendemos que muitas das mudanças sugeridas e esperadas para o ensino da matemática podem surgir de ideias simples agregadas à disposição do professor em encarar novos desafios e tentar experimentar novas metodologias e estratégias diferenciadas.

Nos dias atuais, cada vez mais escolas estão usufruindo de computadores e tecnologias de informação e comunicação. O professor, nesse contexto, deve procurar se preparar para utilizar estes recursos de modo a tirar proveito para ensino e também contribuir como mediador no processo ensino-aprendizagem e garantir que estas estratégias promovam a aprendizagem de novos saberes. Nossa proposta insere-se perfeitamente nesta perspectiva.

Por fim, esperamos que este trabalho possa contribuir, para o ensino da matemática com foco no ensino de coordenadas polares. E além de oferecer aos professores uma nova metodologia para o ensino de coordenadas polares, nossa proposta também visa oportunizar um momento de reflexão sobre a prática pedagógica e a busca de alternativas para a melhoria da educação através da matemática.

\section{REFERÊNCIAS}

ALMEIDA, FJ; Fonseca Junior, F.M. Projetos e ambientes inovadores. Brasília: Secretaria de Educação a Distância - Seed/ Proinfo- Ministério da Educação, 2000.

ACKER, Felipe. Calculo Vetorial e Geometria Analítica: Livro 1: O Plano. Instituto de Matemática, UFRJ, Rio de Janeiro, 2016. Disponível em http://im.ufrj.br/ noseda/2017-1/Livro1.pdf Acesso em 03 de Setembro de 2020. 
BRASIL. Ministério da Educação. Parâmetros Curriculares Nacionais - Ensino Médio. Brasília. Ministério da Educação, 2002.

BORBA, M. C.; PENTEADO, M. G. Informática e Educação Matemática. 3.ed. Belo Horizonte: Autêntica, 2007.

CAMPOS, Márcia de Borba. Construtivismo. Disponível em http://penta.ufrgs.br / marcia/piaget.htm Acesso em 28 de Setembro de 2016.

DUARTE, Sinara. Fazendo as pazes com o bicho papão: A Matemática e o SL. In: Revista Espírito Livre, n.002, p. 74-78, maio. 2009. 89 p.

GERHARDT, T. E. \& SILVEIRA, D. T. Métodos de pesquisa. 1. ed. Porto Alegre: UFRGS, 2009.

GRAVINA, Maria Alice. . [et al.]. Matemática, Mídias Digitais e Didática: tripé para a formação de professores. Porto Alegre: Evangraf, 2012.

MAGEDANZ, Adriana. Computador: Ferramenta de trabalho no Ensino (de Matemática). 2004. 14f. Curso de Pós-Graduação Lato Sensu. Especialização em ensino de Matemática - UNIVATES - Centro Universitário, Lajeado, 2004. Disponível em http://ensino.univates.br/magedanza/pos/artigo_final_adriana_magedanz.pdf Acesso em 29 de Setembro de 2016.

PAPERT, Seymour. A máquina das crianças: repensando a escola na era digital. Porto Alegre: Artes Médicas, 1994.

PIAGET, Jean. Seis estudos de psicologia. Trad. Maria Alice Magalhães DAmorim e Paulo Sérgio Lima Silva. Rio de Janeiro: Forense, 1964.

STAHL, Marimar M. A formação de professores para o uso das novas tecnologias de comunicação e informação. In: CANDAU, Vera Maria (org). Magistério: construção cotidiana. 6 ed. Petrópolis, RJ: Vozes, 2008. p. 292-317. 
SILVA, José Orlando. FERNANDES, Natal Lania Roque. Tecnologias da Informação e Comunicação na Educação de Jovens e Adultos. Disponível em http://portal.mec. go v.br/setec/arquivos/pdf3/tcc_tecnologias.pdf Acesso em 29 de Setembro de 2016.

VALENTE, José Armando (org). O computador na sociedade do conhecimento. Campinas: UNICAMP/NIED, 1999.

VIEIRA, F. (1999) Avaliação de software Educativo: Reflexões para uma análise Criteriosa.

Enviado: Agosto, 2020.

Aprovado: Setembro, 2020. 